\title{
Action Observation and Cortical Connectivity: Evidence from EEG Analysis
}

\author{
Sik-Hyun Kim¹, Jeong-Sun Cho² \\ 'Department of Physical Therapy, Sunlin University, Pohang; ²Department of Lifelong Education Convergence, Daegu Haany University, Gyeongsan, \\ Korea
}

Purpose: The purpose of this study was to examine the changes in electroencephalogram (EEG) coherence and brain wave activity for first-person perspective action observation (1A0) and third-person perspective action observation (3A0) of healthy subjects.

Methods: Thirty healthy subjects participated in this study. EEG was simultaneously recorded during the Relax period, the $1 \mathrm{AO}$, and the $3 \mathrm{AO}$, with event-related desynchronization (ERD) and coherence connectivity process calculations for brain wave (alpha, beta and mu) rhythms in relation to the baseline.

Results: Participants showed increased coherence in beta wave activity in the frontal and central areas $(p<0.05)$, during the $1 \mathrm{AO}$ using right-hand activity. Conversely, the coherence of the alpha wave decreased statistically significantly decreased in the frontocentral and parieto-occipital networks during the observation of the $1 \mathrm{AO}$ and the $3 \mathrm{AO}$. The ERD values were larger than $40 \%$ for both central regions but were slightly higher for the $C 4$ central region. The high relative power of the alpha wave during $1 \mathrm{AO}$ and $3 \mathrm{AO}$ was statistically significantly decreased in the frontal, central, parietal, and occipital regions. However, the relative power of the beta wave during $1 \mathrm{AO}$ and $3 \mathrm{AO}$ was statistically significantly increased in the parietal and occipital regions. Especially during $1 \mathrm{AO}$, the relative power of the beta wave in the $\mathrm{C} 3$ area was statistically significantly increased $(\mathrm{p}<0.05)$.

Conclusion: These findings suggest that $1 \mathrm{AO}$ and $3 \mathrm{AO}$ action observations are relevant to modifications of specific brain wave coherence and ERD values. EEG cortical activity during action observation may contribute to neural reorganization and to adaptive neuroplasticity in clinical intervention.

Keywords: Action observation, Cortical coherence, ERD, Neural plasticity

\section{INTRODUCTION}

Therapeutic approaches to stroke patients emphasize recovery of their motor function. Active physical training for functional improvement of patients during an early phase of stroke is crucial. Intensive motor skill and cognitive interventions during an early phase of stroke are very important to normalize functional activity in the future. Most motor skills are obtained through physical practice, but observation of exercise activities may activate the mirror neuron system, enabling learning of physical exercises and inducing neuroplasticity alterations. ${ }^{1}$ It was identified that in a human adult brain, the area activated in action execution and the area activated during action observation were the same in the neural network sys-

Received Dec 8, 2016 Revised Dec 20, 2016

Accepted Dec 30, 2016

Corresponding author Sik-Hyun Kim

E-mail spritup@sunlin.ac.kr tem called the mirror neuron system. ${ }^{2}$

Despite the clinical importance of repetitive physical interventions, action observation must be used for activation of the brain motor system when practical physical training cannot be performed due to severe physical disability, pain, or excessive abnormal muscle tone. In particular, action observation may be introduced and used as a new therapeutic intervention to overcome learned nonuse resulting from compulsory movement (forced use of the unaffected side), a maladaptive plasticity factor.

Motor recovery should accompany motor learning in the damaged neural network without failure, and action observation intervention should be used as a new treatment paradigm based on the result of motor learning being activated using the action observa-

Copylight @2016 The Korea Society of Physical Therapy

This is an Open Access article distribute under the terms of the Creative Commons Attribution Non-commercial License (Http:// creativecommons.org/license/by-nc/4.o.) which permits unrestricted non-commercial use, distribution, and reproduction in any medium, provided the original work is properly cited. 
tion network. ${ }^{3}$ Accordingly, the purposes of this study are 1) to examine alterations in alpha and beta wave bands according to action observation perspectives, 2) analyze brain coherence and connectivity according to action observation perspectives, and 3) provide a neuroscientific basis for action observation aimed at application to stroke treatment and interventions by analyzing high alpha eventrelated desynchronization (ERD).

\section{METHODS}

\section{Subjects}

Thirty healthy subjects participated in the study (26 women and 4 men; average age $20.73 \pm 1.11$ ). All participants were right-handed, had normal vision and had no history of neurological disorders, and achieved a score $(1.96 \pm 0.38)$ of equal to or less than 2.8 on the Vividness of Movement Imagery Questionnaire (VMIQ), indicating that their imagery ability was at least moderately clear and vivid. All participants were informed about the study protocol before they signed the consent forms.

\section{Experimental procedure}

Before the experiment started, the participants removed metal accessories, sat on a chair, and were secured to the chair with cervical collar and a let to prevent measurement error. To ensure that the participants attended to the video stimuli, they were instructed to pay attention to the hand movements they would see. The participants watched 1 continuous video with 5 intervals: (1) Relax interval 1: fullscreen visual white noise was presented as a baseline condition. This interval lasted 3 minutes; (2) First-person perspective action observation (1AO) interval: 50 set of hand activity with only one right hand on the table as a first person perspective was presented. This interval lasted 2 minutes 30 Seconds; (3) Relax interval 2: full-screen visual white noise was presented to stabilize the brain wave. This interval lasted 1 minutes; (4) Third-person perspective action observation (3AO) interval: 50 set of hand activity with another person's upper body as a third person perspective was presented. This interval lasted 2 minutes 30 Seconds; (5) Relax interval 3: full-screen visual white noise was presented to stabilize the brain wave. This interval lasted 1 minutes. The duration of the total experiment was 10 minutes. One set of hand activity was started with neutral position and organized according to following sequence: 1 Second of supination, 1 Second of pronation, and 1 Second of white screen to wait for the next set. Electroencephalogram (EEG) signals were continuously recorded using the LXE3232-RF (LAXTHA Inc., Korea).

\section{EEG recordings}

The locations of the electrode channels were F3, F4, C3, C4, P3, $\mathrm{P} 4, \mathrm{O} 1$, and $\mathrm{O} 2$, which were placed using the 10-20 system. The reference and ground electrodes were placed on the participants' ear lobes. The experiment took place in a quiet, well-lit room. The EEG data were analyzed by using Telescan (LAXTHA Inc., Korea). The relative band power and coherence were computed following the manufacturer's instructions.

\section{Event-related desynchronization analysis}

ERD is defined as the percentage of decrease (positive ERD) or increase (negative ERD) in band power during a test interval compared with a reference interval. First, the EEG signal from the C3 was band-pass filtered within the mu wave frequency $(8-13 \mathrm{~Hz})$, and then the filtered data were squared and averaged within the test interval $(1 \mathrm{~s})$. Second, the band power was averaged over the number of epochs (50 times). Third, the alterations in band power were calculated using the following formula: ERD $=[$ (band power reference - band power test) / (band power reference) $] \times 100$.

\section{Statistical analysis}

The statistical software R was used to analyze the EEG data. The frequency of the brain wave was selected based on the type: mu wave $(8-13 \mathrm{~Hz})$, high alpha wave $(9.8-12.7 \mathrm{~Hz})$, and beta wave $(13-30$ $\mathrm{Hz}$ ). One-way repeated-measures analysis of variance (ANOVA) was used to evaluate the absolute log ratio and ERD of the mu rhythm and the relative wave power and coherence of the high alpha and beta waves during the Relax period, the $1 \mathrm{AO}$, and the $3 \mathrm{AO}$. When the differences were statistically significant, the Tukey test was applied.

\section{RESULTS}

\section{Suppression of the mu rhythm}

During the $1 \mathrm{AO}$, the absolute mu rhythm log ratio at $\mathrm{C} 3$ was $-0.2295 \pm 0.173(\mathrm{p}<0.0001)$ and at C4 was $-0.2514 \pm 0.2009$ $(\mathrm{p}<0.0001)$. During $3 \mathrm{AO}$, the absolute mu rhythm $\log$ ratio at $\mathrm{C} 3$ 


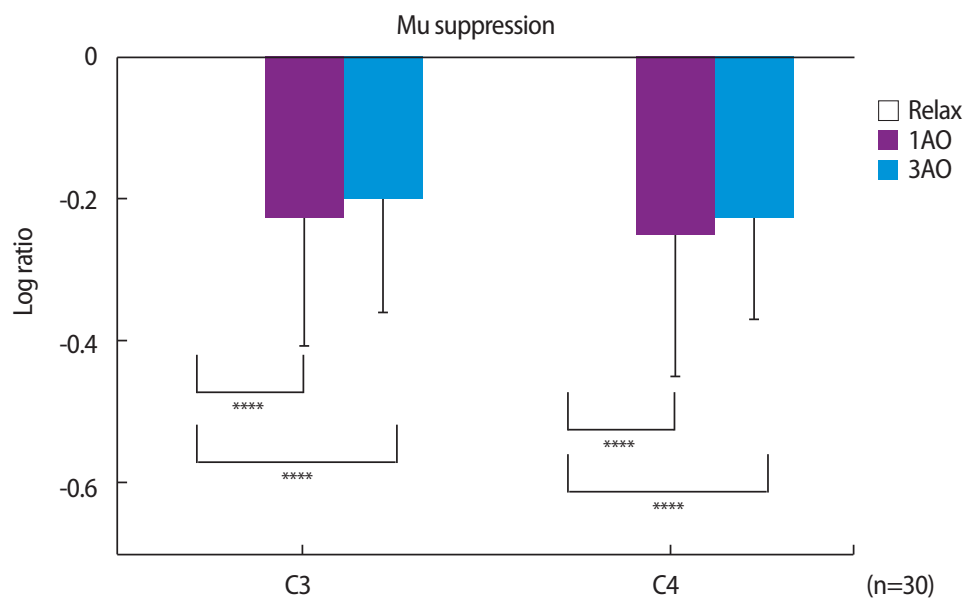

\begin{tabular}{lcccc}
\hline & 1 Person perspective action observation (1AO) & 3 Person perspective action observation (3AO) & $F$ & $P$ \\
\hline C3 & $-0.2295 \pm 0.173$ & $-0.2027 \pm 0.1579$ & 30.53 & $<0.001$ \\
C4 & $-0.2514 \pm 0.2009$ & $-0.2260 \pm 0.1449$ & 31.07 & $<0.001$ \\
\hline
\end{tabular}

Mean \pm standard deviation

Figure 1. Changes in the suppression of the mu rhythm. Bars represent the mean log ratio of power in the mu frequency (8-13 $\mathrm{HZ})$ during the observation of the white screen (Relax, white), the first-person perspective (1AO, purple) video, and the third-person perspective (3AO, blue) video compared with the power in the Relax period for C3 and C4. Error bars represent the standard deviation.

${ }^{* * * *} \mathrm{p}<0.0001 .1 \mathrm{AO}$, first-person perspective action observation; $3 \mathrm{AO}$, third-person perspective action observation.

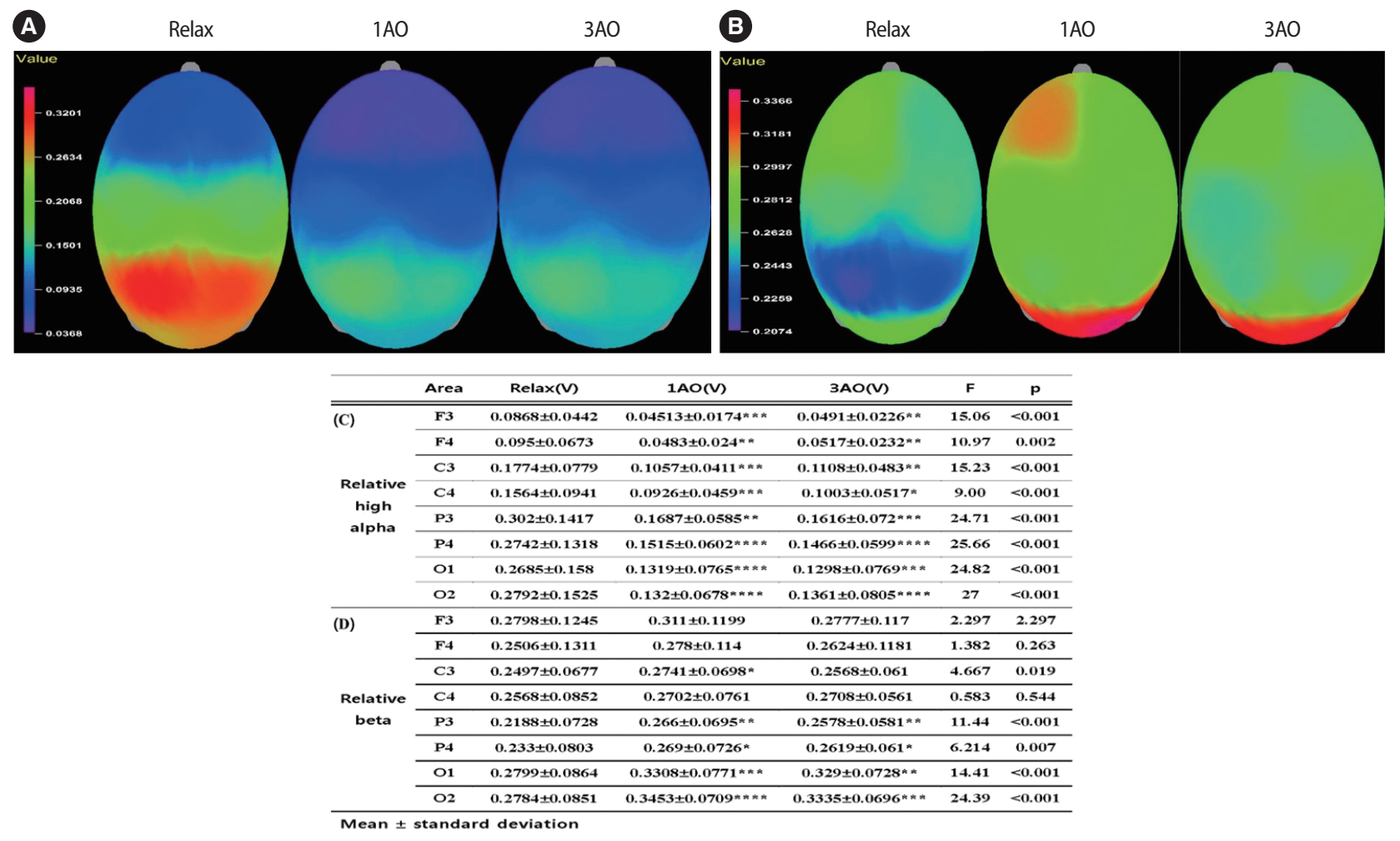

Figure 2. Relative power of the high alpha (C) and beta waves (D). (A) Map of the brain for the relative power of the high alpha (9.8-12.7 Hz) wave. The power of the high alpha wave was statistically significantly decreased in all regions for the first-person perspective action observation $(1 \mathrm{AO})$ and the third-person perspective action observation (3AO) compared with the Relax period. (B) Map of the brain for the relative power of the beta $(13-30 \mathrm{~Hz})$ wave. The power of the beta wave increased statistically significantly $(p<0.05)$ during the $1 \mathrm{AO}$ in the F3 and C3 compared with the Relax period and the $3 \mathrm{AO}$. In the parietal and occipital regions, the relative power of the beta wave increased statistically significantly during both types of observational perspective compared with the Relax period.

${ }^{*} \mathrm{p}<0.05,{ }^{* *} \mathrm{p}<0.01,{ }^{* * *} \mathrm{p}<0.001,{ }^{* * * *} \mathrm{p}<0.0001$. 
was $-0.2027 \pm 0.1579(\mathrm{p}<0.0001)$ and at $\mathrm{C} 4$ was $-0.2260 \pm 0.1449$ $(\mathrm{p}<0.0001$, Figure 1). Compared with the Relax period (the baseline), the mu rhythm during the $1 \mathrm{AO}$ and the $3 \mathrm{AO}$ was statistically significantly suppressed. No statistically significant difference was found between the types of observational perspective.

\section{Relative power of the high alpha and beta waves}

The relative power of the high alpha wave during the $1 \mathrm{AO}$ and the $3 \mathrm{AO}$ decreased statistically significantly in the frontal, central, parietal, and occipital regions compared with the Relax period (Figure $2 \mathrm{~A}, 2 \mathrm{C})$. The relative power of the beta wave during the $1 \mathrm{AO}$ was $0.2741 \pm 0.0698$ in the $\mathrm{C} 3$ region. In the parietal region, the relative power of the beta wave was $0.266 \pm 0.0695(\mathrm{P} 3)$ and $0.269 \pm 0.0726$ (P4) during the $1 \mathrm{AO}$ and $0.2578 \pm 0.0581$ (P3) and $0.2619 \pm 0.061$ (P4) during the $3 \mathrm{AO}$. In the occipital region, the relative power of the beta wave was $0.3308 \pm 0.0771(\mathrm{O} 1)$ and $0.3453 \pm 0.0709(\mathrm{O} 2)$ during the $1 \mathrm{AO}$ and $0.329 \pm 0.0728(\mathrm{O} 1)$ and $0.3335 \pm 0.0696(\mathrm{O} 2)$ during the $3 \mathrm{AO}$. The relative power of the beta wave during the $1 \mathrm{AO}$ and the
$3 \mathrm{AO}$ was statistically significantly increased in the parietal and occipital regions compared with the Relax period. Especially during the $1 \mathrm{AO}$, the relative power of the beta wave in the $\mathrm{C} 3$ area was statistically significantly increased $(\mathrm{p}<0.05$, Figure $2 \mathrm{~B}, 2 \mathrm{D})$.

\section{Coherence of the high alpha and beta waves}

The EEG coherence of the high alpha networks were decreased during the $1 \mathrm{AO}$ and the $3 \mathrm{AO}$ compared with the Relax period. The frontocentral and parieto-occipital networks were decreased during both types of observational perspective (Figure 3A). The interhemispheric networks of all regions were statistically significantly decreased (Figure 3A, 3B).

The EEG coherence of beta networks were increased during the $1 \mathrm{AO}$ and the $3 \mathrm{AO}$ compared with the Relax period. The frontocentral, centroparietal, and parieto-occipital networks was increased during both types of observational perspective. The number of coherence lines increased according to the type of observational perspective, especially $3 \mathrm{AO}$ (Figure 4A). The interhemispheric net-

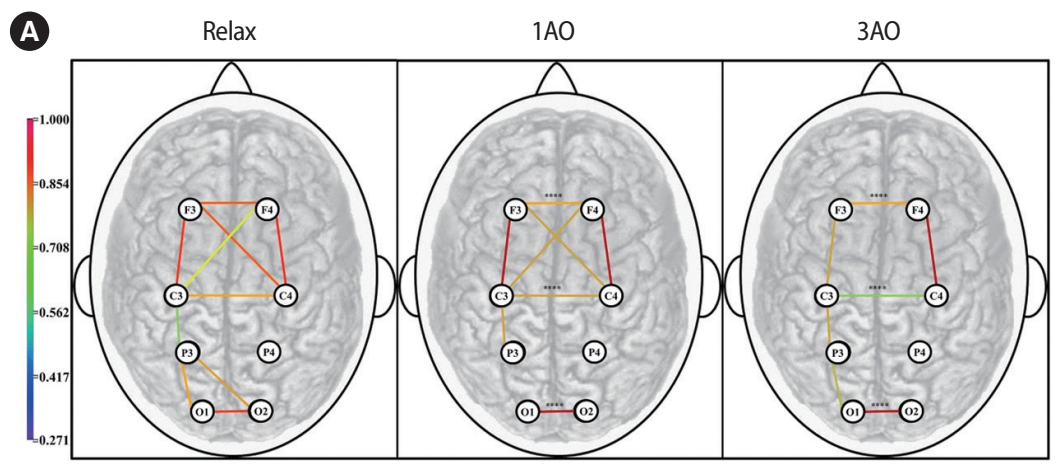

B

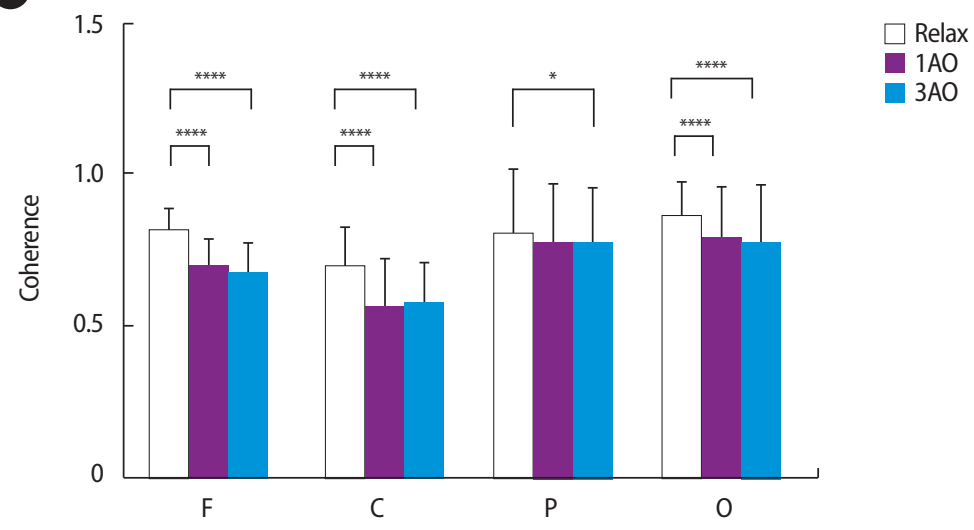

Figure 3. Coherence of the high alpha wave. (A) Map of the brain. Lines represent coherence between linked areas. (B) Coherence of the interhemispheric network. Bars represent the coherence between F3 and F4 (F), C3 and C4 (C), P3 and P4 (P), O1 and O2 (O) in the high alpha frequency during the Relax period (white), the first-person perspective action observation (1AO; purple) and the third-person perspective action observation (3AO; blue). Error bars represent the standard deviation.

${ }^{*} p<0.05,{ }^{* *} p<0.01,{ }^{* * *} p<0.001,{ }^{* * * *} p<0.0001$. 

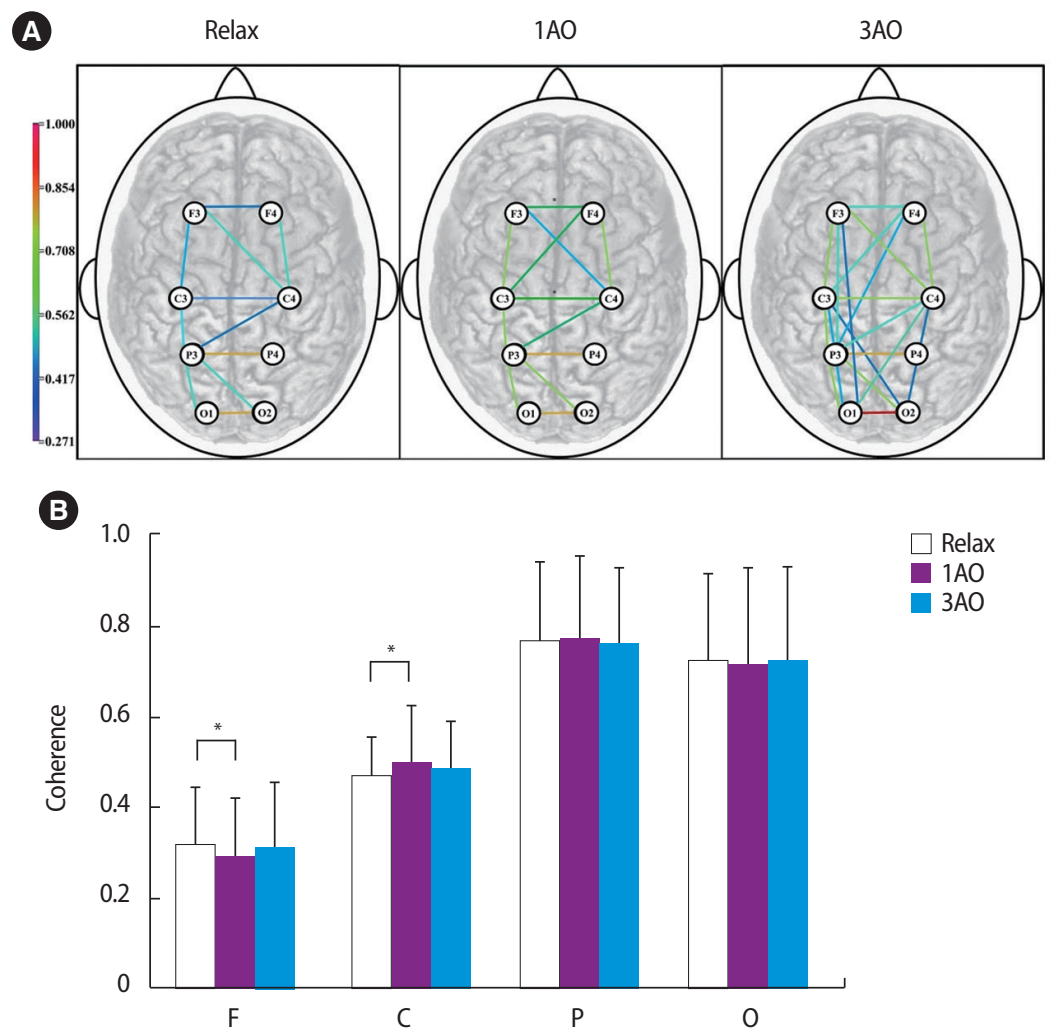

Figure 4. Coherence of the beta wave. (A) Map of the brain. Lines represent coherence between linked areas. (B) Coherence of the interhemispheric network. Bars represent the coherence between F3 and F4 (F), C3 and C4 (C), P3 and P4 (P), and O1 and O2 (O) in the beta frequency during the Relax period (white), the first-person perspective action observation (1AO; purple) and the third-person perspective action observation (3AO; blue). Alterations occurred in the interhemispheric networks in the frontal and central regions were statistically significantly during the $1 \mathrm{AO}$. Error bars represent the standard deviation. ${ }^{*} p<0.05$.

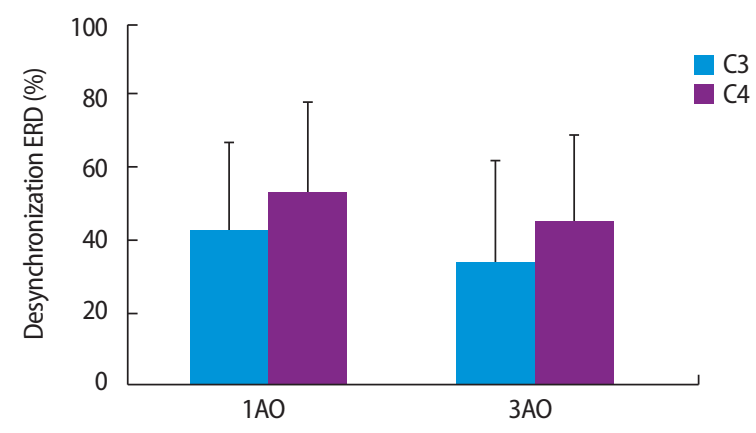

Figure 5. Power associated with ERD of the mu frequency. Bars represent the ERD values in the mu frequency $(8-13 \mathrm{HZ})$ during first-person perspective action observation (1AO) and third-person perspective action observation (3AO) intervals compared with the power in the Relax period for C3 (blue) and C4 (purple). Error bars represent the standard deviation.

works in the frontal and central regions were significantly altered during $1 \mathrm{AO}(\mathrm{p}<0.05$, Figure 4A, 4B).

\section{ERD of the mu wave}

The ERD value for the mu wave indicated that the power of the mu wave was suppressed by action observation. The ERD values were larger than $40 \%$ in both central regions. The ERD value in the $\mathrm{C} 4$ region was slightly higher than that in the $\mathrm{C} 3$ region but had no statistical significance (Figure 5).

\section{DISCUSSION}

Activation of the premotor cortex may be induced by action observation, and the mirror system may be activated by observation of other physical actions, as well as by observation of hand actions. ${ }^{5}$ In the clinical field of physical therapy, improvement in the functional activity of the upper extremity is essential for improvement of activities of daily living. The fact that neuronal recruitment of the mirror neuron system may be activated by other action observations in addition to hand observation means that the application spectrum of therapeutic interventions may be expanded. In particular, therapeutic interventions to induce brain activity, such as action observation before and after treatment in stroke patients with cognitive-execu- 
tive disability-like gait apraxia, may trigger much functional improvement in the patients, which is important as a new therapeutic paradigm. Mirror neurons are activated by action observation but also respond to action-related sounds. ${ }^{6}$ A major factor in decreasing the therapeutic effects of therapeutic approaches is a lack of patient concentration. A therapist provides verbal commands to induce a patient's awakening during clinical treatment intervention. Responsiveness of the mirror system to acoustic stimulation could explain the therapeutic scientific basis for verbal clues in particular when proprioceptive facilitation techniques are applied to the neuromuscular system. Therefore, a therapeutic approach to induce patients' attention and awakening using a neurophysiological mechanism that responds to an action-related sound may be applied for standard improvement in physical activity.

In a study that examined the effects of action observation applied to a child, when goal-directed movements were shown to an 8-month-old infant, there was a statistically significant change in the ERD of the mu rhythm related to direct activation of the motor system. ${ }^{7}$ ERD shows that alterations occur in neurostimulation of the central brain region that suggest motor learning occurs with action observation. The alterations in ERD due to action observation identified in this study of adults in their 20 Seconds also provide the possibility of clinical application. During the first-person perspective and the third-person perspective action observations in this study, there were no statistically significant differences in ERD percentages among the groups, but ERD of the mu rhythm of the C3 and $\mathrm{C} 4$ areas, meaning central brain activation, was identified (Figure 5).

Training of action observation and direct execution of observed activities were applied to stroke patients once per day. The patients' clinical functional scale score improved statistically significantly, and activity of the bilateral ventral premotor cortex, superior temporal gyrus, and supplementary motor area (which are function-related cortical areas) and the contralateral supramarginal gyrus increased statistically significantly. ${ }^{8}$ Alterations in neural activity were identified in the first-person perspective and third-person perspective action observation groups in the present study. In particular, alterations in the relative power of the high alpha rhythm related to semantic memory were statistically significant in all frontal (F3, F4), central (C3, C4), parietal (P3, P4), and occipital (O1, O2) areas (Figure $2 \mathrm{~A}, 2 \mathrm{C}$ ). The relative power of the beta rhythm closely related to conscious activities was observed in the $\mathrm{C} 3, \mathrm{P} 3, \mathrm{P} 4, \mathrm{O} 1$, and $\mathrm{O} 2$ areas in the $1 \mathrm{AO}$ group (Figure 2B, 2D). This result was statistically significant according to the results for the coherence of the beta wave (Figure 4).

During action observation, alterations in the coherence of the beta wave were identified only in the frontal areas and the central region of the $1 \mathrm{AO}$ group. Thus, the first-person perspective could be used as a treatment tool for improvement in functional activities used in physical therapy. Mirror neurons directly respond to visual representation, such as action observation, and to acoustic stimuli of sounds being made when movements are performed. Such response characteristics were identified in a study in which motor facilitation induced by action observation was analyzed. ${ }^{9}$ As a new clinical proposal, an action observation device that combines virtual reality and a haptic sensor should be developed for therapeutic interventions to provide observation stimulation and acoustic stimulation simultaneously.

During action observation, excitability of the primary motor cortex (M1) increases with a characteristic increase in the motor evoked potentials (MEPs) of the agonist muscle. ${ }^{10}$ The statistical significance of the suppression of the mu wave in the central C3 and C4 regions in this study suggest an association between the excitability of the motor cortex and MEPs (Figure 1). To sum up the previous study and the present study, action observation has a clinical therapeutic effect of stimulating neural activation of the primary motor cortex and engaging in actual exercise execution as suppression of the mu rhythm.

According to the results of various EEG studies, the EEG frequency wave band represents brain activity, and characteristically alpha $(8-12 \mathrm{~Hz})$ and beta $(14-30 \mathrm{~Hz})$ bands were found to be EEG waves largely occurring during voluntary motor activity or somatosensory processing. ${ }^{11}$ In the present study, alterations in the alpha and beta rhythms according to the observation perspectives were examined, and statistically significant statistical results during the $1 \mathrm{AO}$ and $3 \mathrm{AO}$ action observations were found. In particular, statistically significant alterations in the coherence values of the brain waves that can explain functional connectivity were identified in the frontal and central beta bands. These results show an association between neuroplasticity and neural network reorganization in accordance with action observation.

ERD among EEG waves is an indicator that represents brain cor- 
tical activity or arousal states. ERD tends to decrease when a subject performs an action. In relation to action observation, the most sensitive ERD responses occur in the C3 and C4 regions, which are central regions in the brain. ${ }^{12}$ As a very exciting study result in clinical therapeutic terms, more powerful ERD of the alpha wave appeared during observation of transitive activity, more object-directed activity than simple activity. ${ }^{13}$ In a study by Gonzalez-Rosa et al., ${ }^{14}$ the ERD of the alpha wave of the action observation group had the highest reactivity in the frontocentral and bilateral parietal areas, and ERD of the beta wave appeared in the parietal lobe areas during actual physical motor performance. In particular, compared to the control group, the action observation group exhibited better adaptability when they performed or learned novel motor tasks. They reported that action observation was a very effective intervention during the early motor learning phase. These results suggest the possibility of motor learning related to action observation, and the outcome of specific ERD/ERS activation of the frontocentral areas and parietal areas, forming a functional network, is evidence of the action observation learning process.

During action observation, ERD of the alpha and mu rhythms mainly appear in the inferior parietal cortex, frontal cortex, supplementary motor area, and dorsal premotor cortex..$^{15}$ Beta wave activity increases during task execution, and as the precision of the motor tasks performed increases, the synchrony of the beta waves increases. ${ }^{16}$ In the present study as well, statistically significant alterations in the alpha and beta wave bands were observed for each observation perspective. These alterations provide a strong therapeutic ground for action observation intervention in clinical therapeutic terms compared with previous results that such alterations affect the precision of motor tasks and motor learning.

Action observation and motor memory formation was studied in chronic stroke patients using transcranial magnetic stimulation (TMS). The corticomotor activity changed in the hand representation areas of the primary motor areas in the action observation group. ${ }^{17}$ Neurophysiologically, activity in the primary motor cortex increases during the explicit motor learning phase and in particular, directly engages in the velocity-dependency learning process. Therapeutic interventions for stroke patients to overcome functional disability are the most important factor in physical therapy in clinical terms. Action observation is a very effective tool aimed at improving the performance of specific physical motor skills to enhance stroke patients' physical disability. ${ }^{18}$ Action observation is a very useful method as a therapeutic tool to promote formation of motor memory trace and motor learning. ${ }^{19}$

Bassolino et al., ${ }^{20}$ study of TMS showed interesting results in relation to physical therapy clinical intervention. They immobilized the right arm of a subject and conducted motor imagery and action observation of constrained limb movements. The researchers showed that a clear recruitment curve for neural activity and the MEP occurred in the hand corticomotor representation areas of the action observation group only. Among the factors affecting maladaptive plasticity, compensatory movement and competitive interaction decrease ipsilesional hemisphere activity and restrict the functional activity of the affected (paretic) side. In addition, it induces interhemispheric imbalance into abnormal motor patterns. To sum up the results of the prior research by Bassolino et al. ${ }^{20}$ and the present study, action observation is a clinical therapeutic intervention that enables overcoming of learned nonuse of patients and induces functional cortex connectivity and accordingly adaptive-experience dependent plasticity.

Motor imagery has many limitations in clinical application. First, a patient must be able to imagine specific actions. Second, it is difficult to clearly identify whether mental training is modified during imagination and progression, in other words, whether the patient properly executes the presented imagination. Nonetheless, action observation intervention may easily apply task-oriented movement or goal-oriented movement by simplifying them and may induce adaptive neuroplasticity.

In a previous experiment, action observation intervention was applied as a method for treating upper limb motor function of middle cerebral cortex ischemic stroke patients, the motor function of the experimental group to which action observation interventional treatment was applied for 4 weeks improved statistically significantly. In addition, according to the functional magnetic resonance imaging (fMRI) of the action observation group, the neural activity of the bilateral ventral premotor cortex, bilateral superior temporal gyrus, supplementary motor area, and contralateral supramarginal gyrus increased statistically significantly. ${ }^{8}$ These research results are meaningful when they are considered in association with the statistically statistically significant outcome of the coherence data for alpha and beta waves identified in the frontocentral region (F3, F4; $\mathrm{C} 3, \mathrm{C} 4)$ and parieto-occipital areas (P3, P4; O1, O2) in the present 
study.

The basal ganglia that play an important role in motor planning and learning are very importantly connected with the mirror neuron system in anatomical terms. ${ }^{21}$

During unimanual action observation, functional network areas, including the occipito-temporal, parietal, and premotor cortex, are activated. ${ }^{22}$ During bimanual activity observation, bilateral brain activity was identified, and activity of the parietal cortex was prevalent. ${ }^{23}$ This result suggests bimanual band action observation networks may positively affect balanced interhemispheric cortical activity.

According to a study by Garrison et al. ${ }^{24}$ ipsilesional cortical motor activity increased during action observation using the right hand (paretic side) in a patient with left hemiplegia resulting from a stroke. Action observation was applied to the clinical treatment intervention of physical therapy for motor relearning, neural reorganization, and adaptive neuroplasticity.

When brain activation was examined using fMRI according to observational perspectives with right-handed participants as subjects, the parietal cortex of the contralateral hemisphere was activated when the tool-grasping action of the first-person perspective was observed. However, during the third-person perspective action observation, the ipsilateral parietal cortex was activated. ${ }^{25}$ In the present study, when right-hand action observation was applied to righthanded participants, the alterations in the rhythm of the beta wave of the first-person perspective group were statistically significant in the $\mathrm{C} 3, \mathrm{P} 3$, and $\mathrm{O} 1$ regions and in the ipsilateral $\mathrm{P} 4$ and $\mathrm{O} 2$ regions. In the case of the third-person perspective observation group, there was a statistically significant alteration in the contralateral P3 and $\mathrm{O} 1$ regions and the ipsilateral $\mathrm{P} 4$ and $\mathrm{O} 2$ regions. This result showed brain connectivity by action observation and may show a relation between interhemispheric activation and intrahemispheric interaction. Maeda et al. ${ }^{26}$ identified that when right-handed action was observed, alterations in brain activation according to the observer's perspective were studied, and the group that observed actions with the first-person perspective had more strongly induced facilitation of the MEP. This result suggests that corticospinal excitability could be considered according to the observer's perspective of action observation.

Alpha wave rhythms generally decrease when actual action is performed ${ }^{27}$ and decrease by indirect cognitive training, such as ac- tion observation. ${ }^{28}$ EEG beta rhythms are reported to originate from the sensory and motor cortexes. ${ }^{29}$ In particular, beta waves respond very sensitively to somatosensory variation in an observed action. ${ }^{30}$

Interhemispheric imbalance is a key factor in functional impairment after a stroke. ${ }^{31}$ In relation to the poor motor recovery of stroke patients, when interhemispheric inhibition increases from the contralesional motor cortex (the unaffected hemisphere) to the ipsilesional motor cortex (the affected hemisphere), the excitability of the ipsilesional motor cortex decreases with functional loss of the paretic extremity.

Interhemispheric inhibition has a wave characteristic of extensive ranges at 6 to $50 \mathrm{~ms}$ and is mediated through the corpus callosum. Clinically, the loss of interhemispheric inhibition in ischemic stroke patient $^{32}$ results in late recovery of motor functions and the occurrence of maladaptive plasticity. This represents abnormal neuroplasticity by compensatory movement (the forced use of the unaffected extremity) and competitive interaction (interhemispheric imbalance). According to research reported by James et al., ${ }^{33}$ who studied connectivity of the brain, an improvement in motor performance was closely related to an increase in interhemispheric communication. Bilateral movement decreases intracortical inhibition in the primary motor cortex and interhemispheric inhibition between the contralesional motor cortex (the unaffected hemisphere) and the ipsilesional motor cortex (the affected hemisphere), promoting corticospinal excitability from the ipsilesional primary motor cortex. $^{34,35}$

Coherence analysis is very important as an indicator of functional recovery among different cortical areas. ${ }^{36}$ Desynchronization of the high alpha wave is closely related to semantic memory performance. ${ }^{37}$ The ERD of the high alpha wave increases statistically significantly when semantic judgment tasks are performed. ${ }^{38}$ Good memory performance may be expressed as a statistically significantly large ERD percentage (\%). Babiloni et al. ${ }^{39}$ reported for a study on EEG coherence that frontoparietal connectivity was considered to contribute to optimizing representational memory during short-term memory. This result suggests an association between the visual process and motor memory when it is compared with the coherence of the beta wave in the present study. Therefore, observational learning is thought to be closely related to frontoparietal connectivity. It was reported that during action observation, interhemi- 
spheric inhibition was dynamically regulated. This could be the result of sensory-motor experience. ${ }^{40}$

To sum up the present results, action observation was shown to have sufficient possibility as a clinically useful treatment tool for stroke. In particular, this intervention is perfect as an important therapeutic intervention to overcome connectivity of the brain and maladaptive neuroplasticity, inducing excitability of the brain and interhemispheric balance by applying the tool before and after a clinical therapeutic intervention. When there are problems, such as limited treatment time, space, and environment, virtual reality-dependent action observation should be applied using smart devices. The present study involved alterations in connectivity of the brain and alpha and beta waves based on action observation of the observer's perspective with healthy individuals as the subjects. Future research should investigate clinical therapeutic interventions by studying alterations in brain waves according to action observation with stroke patients as subjects and by systematically studying the effects of visuosensory observational input on neuroplasticity and motor learning.

\section{REFERENCES}

1. Buccino G. Action observation treatment: a novel tool in neurorehabilitation. Phil Trans R Soc B 2014;369(1644):20130185.

2. Molenberghs P, Cunnington R, Mattingley JB. Brain regions with mirror properties: a meta-analysis of human fMRI studies. Neurosci Biobehav Rev. 2012;36(1):341-9.

3. Buccino G, Vogt S, Ritzl A et al. Neural circuits underlying imitation learning of hand actions: an event-related fMRI study. Neuron. 2004; 42(2):323-34.

4. Klimesch W, Doppelmayr M, Pachinger T et al. Brain oscillations and human memory: EEG correlates in the upper alpha and theta band. Neurosci Lett. 1997;238(1-2):9-12.

5. Buccino G, Binkofski F, Fink GR et al. Action observation activates premotor and parietal areas in a somatotopic manner: an fMRI study. Eur J Neurosci. 2001;13(2):400-4.

6. Kilner JM, Frith CD. A possible role for primary motor cortex during action observation. Proc Natl Acad Sci U S A. 2007;104(21)8683-4.

7. Marshall PJ, Meltzoff AN. Neural mirroring mechanisms and imitation in human infants. Phil Trans R Soc B Biol Sci. 2014;369(1644): 20130620.

8. Ertelt D, Small S, Solodkin A et al. Action observation has a positive impact on rehabilitation of motor deficits after stroke. NeuroImage. 2007;36 Suppl 2:T164-73.

9. McGarry LM, Russo FA, Schalles MD et al. Audio-visual facilitation of mu rhythm. Exp Brain Res. 2012;218(4):527-38.

10. Gangitano M, Mottaghy FM, Pascual-Leone A. Phase-specific modula- tion of cortical motor output during movement observation. NeuroReport. 2001;12(7):1489-92.

11. Neuper C, Pfurtscheller G. Event-related dynamics of cortical rhythms: requency-specific features and functional correlates. Int J Psychophysiol. 2001;43(1):41-58.

12. Babiloni C, Babiloni F, Carducci F et al. Human cortical electroencephalography (EEG) rhythms during the observa-tion of simple aimless movements: a high-resolution EEG study. NeuroImage. 2002;17(2):55972 .

13. Muthukumaraswamy SD, Johnson BW, McNair NA. Mu rhythm modulation during observation of an object-directed grasp. Brain Res Cogn Brain Res. 2004;19(2):195-201.

14. Gonzalez-Rosa JJ, Natali F, Tettamanti A et al. Action observation and motor imagery in performance of complex movements: Evidence from EEG and kinematics analysis. Behav Brain Res. 2015;281:290-300.

15. Arnstein D, Cui F, Keysers C et al. Suppression during action observation and execution correlates with BOLD in dorsal premotor, inferior parietal, and SI cortices. J Neurosci. 2011;31(40):14243-9.

16. Kristeva-Feige R, Fritsch C, Timmer J et al. Effects of attention and precision of exerted force on beta range EEG-EMG synchronization during a maintained motor contraction task. Clin Neurophysiol. 2002;113(1): 124-31.

17. Celnik P, Webster B, Glasser DM et al. Effects of action observation on physical training after stroke. Stroke. 2008;39(6):1814-20.

18. Vogt S, Thomaschke R. From visuo-motor interactions to imitation learning: behavioural and brain imaging studies. J. Sports Sci. 2007; 25(5):497-517.

19. Celnik P, Webster B, Glasser DM et al. Effects of action observation on physical training after stroke. Stroke. 2008;39(6):1814-20.

20. Bassolino M, Campanella M, Bove M et al. In press. Training the motor cortex by observing the actions of others during immobilization. Cereb Cortex. 2013;24(12):3268-76.

21. Doyon J, Bellec P, Amsel R et al. Contribution of the basal ganglia and functionally related brain structures to motor learning. Behav Brain Res. 2009;199(1):61-75.

22. Grosbras MH, Beaton S, Eickhoff SB. Brain regions involved in human movement perception: a quantitative voxel-based meta-analysis. Hum Brain Mapp. 2012;(33):431-54.

23. Heitger MH , Macé M JM., Jastorff J et al. Cortical regions involved in the observation of bimanual actions. J Neurophysiol. 2012;108(9):2594611.

24. Garrison KA, Aziz-Zadeh L, Wong SW et al. Modulating the motor system by action observation after stroke. Stroke. 2013;44(8):2247-53.

25 Vingerhoets G, Stevens L, Meesdom M et al. Influence of perspective on the neural correlates of motor resonance during natural action observation. Neuropsychol Rehabil. 2012;22(5):752-67.

26. Maeda F, Kleiner-Fisman G, Pascual-Leone A. Motor facilitation while observing hand actions: specificity of the effect and role of observer's orientation. J Neurophysiol. 2002;87(3):1329-35.

27. Neuper C, Wörtz M, Pfurtscheller G. ERD/ERS patterns reflecting sensorimotor activation and deactivation. Prog Brain Res. 2006;159:211-22.

28. Avanzini P, Fabbri-Destro M, Dalla Volta R et al. The dynamics of sensorimotor cortical oscillations during the observation of hand movements: an EEG study. PLoS One. 2012;7(5):e37534. 
29. Gaetz W, Cheyne D. Localization of sensorimotor cortical rhythms induced by tactile stimulation using spatially filtered MEG. Neuroimage. 2006;30(3):899-908

30. Jensen O, Goel P, Kopell N et al. On the human sensorimotor-cortex beta rhythm: Sources and modeling. Neuroimage. 2005;26(2):347-55.

31. Auriat AM, Neva JL, Peters S et al. A review of transcranial magnetic stimulation and multimodal neuroimaging to characterize post-stroke neuroplasticity. Front Neurol. 2015;6:226.

32. Boroojerdi B, Diefenbach K, Ferbert A. Transcallosal inhibition in cortical and subcortical cerebral vascular lesions. J Neurol Sci. 1996;144(14):160-70.

33. James GA, Lu ZL, VanMeter JW et al. Changes in resting state effective connectivity in the motor network following rehabilitation of upper extremity poststroke paresis. Top Stroke Rehabil. 2009;16(4):270-81.

34. Stinear JW, Byblow WD. Rhythmic bilateral movement training modulates corticomotor excitability and enhances upper limb motricity poststroke: a pilot study. J Clin Neurophysiol. 2004;21(2):124-31.
35. Stinear JW, Byblow WD. Disinhibition in the human motor cortex is enhanced by synchronous upper limb movements. J Physiol. 2002;543(Pt 1):307-16.

36. Andrew C, Pfurtscheller G. Event-related coherence as a tool for studying dynamic interaction of brain regions. Electroencephalogr Clin Neurophysiol. 1996;98(2):144-8.

37. Klimesch W, Schimke H, Schwaiger J. Episodic and semantic memory: an analysis in the EEG-theta and alpha band. Electroencephalogr Clin Neurophysiol. 1994;91(6):428-41.

38. Klimesch W, Doppelmayr M, Pachinger T. Brain oscillations and human memory performance: EEG correlates in the upper alpha and theta bands. Neurosci Lett. 1997;238(1-2):9-12.

39. Babiloni C, Carducci F, Vecchio F et al. Functional frontoparietal connectivity during short-term memory as revealed by high-resolution EEG coherence analysis. Behav Neurosci. 2004;118(4):687-97.

40. Gueugneau N, Bove M, Ballay Y et al. Interhemispheric inhibition is dynamically regulated during action observation. Cortex. 2016;78:138-49. 